\title{
Front Matter: Volume 11190
}

, "Front Matter: Volume 11190," Proc. SPIE 11190, Optics in Health Care and Biomedical Optics IX, 1119001 (24 December 2019); doi: 10.1117/12.2560562

SPIE. Event: SPIE/COS Photonics Asia, 2019, Hangzhou, China 


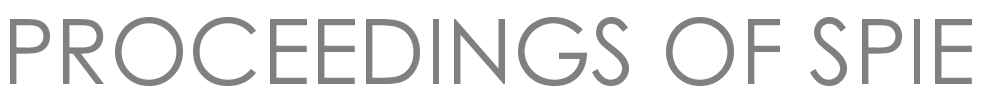

\title{
Optics in Health Care and Biomedical Optics IX
}

\author{
Qingming Luo \\ Xingde Li \\ Ying Gu \\ Yuguo Tang \\ Dan Zhu \\ Editors
}

\section{1-23 October 2019 \\ Hangzhou, China}

Sponsored by

SPIE

cos-Chinese Optical Society

\section{Cooperating Organizations}

Tsinghua University (China) • Peking University (China) • University of Science and Technology of China (China) • Zhejiang University (China) - Tianjin University (China) - Beijing Institute of Technology (China) • Beijing University of Posts and Telecommunications (China) - Nankai University (China) - Changchun University of Science and Technology (China) • University of Shanghai for Science and Technology (China) • Capital Normal University (China) • Huazhong University of Science and Technology (China) - Beijing Jiaotong University (China) • China Jiliang University (China) - Shanghai Institute of Optics and Fine Mechanics, CAS (China) - Changchun Institute of Optics, Fine Mechanics and Physics, CAS (China) • Institute of Semiconductors, CAS (China) • Institute of Optics and Electronics, CAS (China) • Institute of Physics, CAS (China) - Shanghai Institute of Technical Physics, CAS (China) • China Instrument and Control Society (China) • Japan Optical Society (Japan) • Korea Optical Society (Korea, Republic of) • Australia Optical Society (Australia) • Singapore Optical Society (Singapore) • European Optical Society

Supporting Organizations

China Association for Science and Technology (CAST) (China)

Department of Information of National Nature Science Foundation, China (NSFC) (China)

Published by

SPIE

Volume 11190 
The papers in this volume were part of the technical conference cited on the cover and title page. Papers were selected and subject to review by the editors and conference program committee. Some conference presentations may not be available for publication. Additional papers and presentation recordings may be available online in the SPIE Digital Library at SPIEDigitallibrary.org.

The papers reflect the work and thoughts of the authors and are published herein as submitted. The publisher is not responsible for the validity of the information or for any outcomes resulting from reliance thereon.

Please use the following format to cite material from these proceedings:

Author(s), "Title of Paper," in Optics in Health Care and Biomedical Optics IX, edited by Qingming Luo, Xingde Li, Ying Gu, Yuguo Tang, Dan Zhu, Proceedings of SPIE Vol. 11190 (SPIE, Bellingham, WA, 2019) Seven-digit Article CID Number.

ISSN: 0277-786X

ISSN: 1996-756X (electronic)

ISBN: 9781510630970

ISBN: 9781510630987 (electronic)

Published by

SPIE

P.O. Box 10, Bellingham, Washington 98227-0010 USA

Telephone +1 3606763290 (Pacific Time) · Fax +1 3606471445

SPIE.org

Copyright @ 2019, Society of Photo-Optical Instrumentation Engineers.

Copying of material in this book for internal or personal use, or for the internal or personal use of specific clients, beyond the fair use provisions granted by the U.S. Copyright Law is authorized by SPIE subject to payment of copying fees. The Transactional Reporting Service base fee for this volume is $\$ 21.00$ per article (or portion thereof), which should be paid directly to the Copyright Clearance Center (CCC), 222 Rosewood Drive, Danvers, MA 01923. Payment may also be made electronically through CCC Online at copyright.com. Other copying for republication, resale, advertising or promotion, or any form of systematic or multiple reproduction of any material in this book is prohibited except with permission in writing from the publisher. The CCC fee code is $0277-$ $786 \times / 19 / \$ 21.00$.

Printed in the United States of America by Curran Associates, Inc., under license from SPIE.

Publication of record for individual papers is online in the SPIE Digital Library.

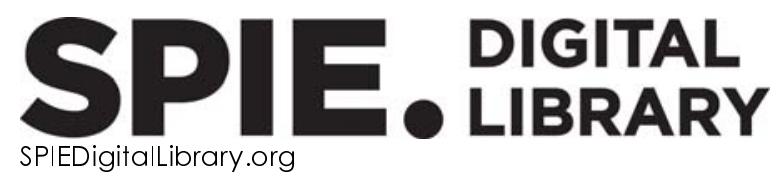

Paper Numbering: Proceedings of SPIE follow an e-First publication model. A unique citation identifier (CID) number is assigned to each article at the time of publication. Utilization of CIDs allows articles to be fully citable as soon as they are published online, and connects the same identifier to all online and print versions of the publication. SPIE uses a seven-digit CID article numbering system structured as follows:

- The first five digits correspond to the SPIE volume number.

- The last two digits indicate publication order within the volume using a Base 36 numbering system employing both numerals and letters. These two-number sets start with 00, 01, 02, 03, 04, 05, 06, 07, 08, 09, 0A, OB ... 0Z, followed by 10-1Z, 20-2Z, etc. The CID Number appears on each page of the manuscript. 


\title{
Contents
}

\author{
ix Authors \\ xii Symposium Committees \\ xviii Conference Committee
}

TRANSLATIONAL OPTICAL TECHNIQUES FOR CLINICAL MEDICINE I

1119006 Ratiometric autofluorescence lifetime imaging system standardization and application for head and neck cancer [11190-4]

TRANSLATIONAL OPTICAL TECHNIQUES FOR CLINICAL MEDICINE II

1119008 Research on automatic identification based on IVOCT images of coronary plaque (Invited Paper) [1 $1190-6]$

\section{NANOBIOPHOTONICS AND SENSORS}

$11190 \mathrm{OH} \quad$ Multiplexing of distributed temperature sensing achieved by nanoparticle-doped fibers [11190-16]

111900 ol Development of robust fiber laser source based on parametric frequency conversion for use in CARS microscopy [1 $11190-17]$

\section{BIOMEDICAL SPECTROSCOPY}

$111900 \mathrm{~J} \quad$ Label-free imaging of lymph nodes with stimulated Raman scattering microscopy [1 $1190-18$ ]

11190 OK Single human platelet study using surface-enhanced Raman spectroscopy as a perspective tool for antiplatelet therapy effectiveness prediction [1 1 190-19]

11190 ON Surface-enhanced Raman spectroscopy of degranulation response to C48/80 in mast cells [11190-22] 
11190 OR Imaging depth extension of optical coherence tomography by optical clearing method in vitro rabbit eye [1 11 190-26]

11190 OU 3-D super-resolution localization microscopy using deep-learning method [1 $1190-29]$

11190 OW Beam-shifting optical coherence tomography for speckle reduction and flow rate measurement [1 11190-31]

11190 OY Fan-shaped tracker (FsT) for particle trajectory reconstruction [1 1190-33]

\section{OPTICAL THERANOSTICS I}

1119013 Two-photon excitation fluorescence lifetime imaging microscopy and spectroscopy for cancer detection [1 11 190-38]

1119015 The heavy atom effect on thiadiazolo[3,4-g]quinoxaline derivatives as novel photosensitizers for photodynamic therapy [1 1190-40]

\section{OPTICAL THERANOSTICS II}

1119016 Preliminary study of sonodynamic effects of a novel water soluble chlorin [1 $1190-41]$

1119019 Optical regulation of stem-cell differentiation by femtosecond-laser photostimulation [1 $1190-44]$

$111901 \mathrm{~A} \quad$ LED-based portable light source for photodynamic therapy [1 1190-45]

TISSUE OPTICS/LASER-TISSUE INTERACTION

$111901 \mathrm{C}$ Simulation of near-infrared light propagation through the thorax of a neonate: addressing the optimisation of source and detector positions for measuring lung oxygen content in preterm infants [11 190-47]

\section{MULTIMODAL BIOMEDICAL IMAGING}

1119016 Toward artifact-free reconstruction of photoacoustic computed tomography images [11190-51]

$111901 \mathrm{~N} \quad$ Ultrahigh-resolution stimulation by femtosecond laser reveals existence and regulation mechanism of nuclear $\mathrm{Ca}^{2+}$ store [1 $1190-58$ ] 
$111901 R \quad$ Computer-aided classification system for early endometrial cancer of co-registered photoacoustic and ultrasonic signals [1 11 190-63]

11190 is Fluorescence spectroscopy study of protoporphyrin IX in tissue-like phantoms [1 $1190-64]$

$111901 \mathrm{~T} \quad$ Automatic detection of leukemia cells by 2D light scattering microfluidic cytometry and deep learning [1 11 190-65]

$111901 \mathrm{U}$ A cost-effective time-gated fluorescence imaging system and its bioimaging applications [11190-66]

$111901 \mathrm{~V} \quad$ High-speed intravascular photoacoustic imaging with blood flushing [1 $1190-68$ ]

$111901 \mathrm{~W} \quad$ Model-based back-projection method in photoacoustic tomography for improved tangential resolution [1 11 190-69]

$111901 \mathrm{X}$ Continuous infrared laser irradiation decreased membrane capacitance of neuron cell [11190-70]

$111901 Y \quad$ Rapid cell phase imaging using quantitative interferometric microscopy with fast shearing phase unwrapping method [1 $11190-71]$

1119012 Study on polarization distribution characteristics of polarized light in scattering media [11190-72]

1119022 Determination of photoacoustic glucose characteristic wavelengths based on synergy interval partial least square algorithm [1 1190-75]

1119024 Label-free discrimination of hepatoma cells based on Raman spectroscopy and multivariate statistical algorithms [1 11 190-77]

1119025 Calibration method of spectral domain OCT system based on characteristic wavelength of light source [1 $1190-78]$

1119026 Swept source polarization-sensitive OCT with fiber-based polarization-diversity detection unit [11190-79]

$111902 \mathrm{~A} \quad$ Label-free light scattering microfluidic cytometry using dual-channel 3D hydrodynamic focusing [1 $11190-83]$

$111902 B \quad$ Determination of cancer cells induced by secretagogues based on fluorescence resonance energy transfer [1 $1190-84]$

$111902 \mathrm{D}$ Simulation of spatial and temporal distribution of singlet oxygen in port wine stain during vascular targeted photodynamic therapy [1 1 1 190-86] 
$111902 \mathrm{~F} \quad$ Non-invasive 3D real time observation of physiological traits during the embryonic development of insects [1 $11190-88]$

$111902 \mathrm{G} \quad$ Photoacoustic signal classification for in vivo photoacoustic flow cytometry based on support vector machine [1 $11190-89]$

$1119021 \quad$ Study on processing of infrared thermogram of ankle soft tissue injury with LDA based on DCT transform [1 11 190-91]

11190 2J Graphene oxide based FRET probe for mast cell degranulation [1 $1190-92]$

$111902 \mathrm{~K} \quad$ Super-resolution ultrasound imaging implemented by SOFI in radio-frequency domain [1 11 190-93]

$111902 \mathrm{~L} \quad$ In vitro study of myocardial fiber structure imaging by Mueller optical coherence tomography [11190-94]

$111902 \mathrm{M} \quad$ Super-resolution x-ray luminescence optical tomography imaging [1 $1190-95]$

$111902 \mathrm{~N} \quad$ Identification of macrophages in breast tumor microenvironment using label-free multiphoton microscopy [1 $11190-96]$

1119020 Multiphoton imaging of normal breast lobules, pleomorphic invasive lobular carcinoma, and classic invasive lobular carcinoma [1 $11190-97]$

$111902 \mathrm{Q} \quad$ Multiphoton imaging of perineural invasion in breast cancer [1 1190-99]

$111902 R \quad$ Space-time adaptive precision imaging [11190-100]

$111902 \mathrm{~T} \quad$ Carotid atherosclerosis detection using photoacoustic imaging system [1 $1190-102]$

$111902 \mathrm{U} \quad$ Identifying pulmonary Cryptococcus neoformans infection by serum surface-enhanced Raman spectroscopy [11190-103]

$111902 \mathrm{~V} \quad$ Application of fluorescence lifetime imaging in skin cancer diagnosis [11190-104]

$111902 \mathrm{X} \quad$ Detection of singlet oxygen luminescence in skin phantom based on optical fiber detection [11 190-106]

1119022 Characterization of human coronary atherosclerotic plaque using spectrum- and timeresolved multiphoton microscopy [11190-108]

1119032 Research on ischemia-reperfusion injury of rat kidney using optical coherence tomography [11190-111]

1119037 DDeep3M-based neuronal cell counting in 2D large-scale images [11 190-117]

1119038 Finite element modeling of mechanical properties of cancer cells [1 $1190-118]$ 
11190 3A UV-LED device effectively increases serum levels of 25(OH)D3 in osteoporosis rats [1 1190-120]

11190 3C Laser speckle correlation imaging with optical clearance for blood flows [1 $1190-122]$

11190 3D Tracking of intracellular doxorubicin-Cu complexes with FLIM technique [1 $1190-123]$

$111903 \mathrm{E} \quad$ Compressed sensing with a novel sparse-sampled camera for spectral domain optical coherence tomography [11190-124]

$111903 \mathrm{~K} \quad$ A fast reconstruction method for super-resolution localization microscopy with gOMP [1 11 190-130] 
Proc. of SPIE Vol. $111901119001-8$

Downloaded From: https://www.spiedigitallibrary.org/conference-proceedings-of-spie on 26 Apr 2023 Terms of Use: https://www.spiedigitallibrary.org/terms-of-use 


\title{
Authors
}

Numbers in the index correspond to the last two digits of the seven-digit citation identifier (CID) article numbering system used in Proceedings of SPIE. The first five digits reflect the volume number. Base 36 numbering is employed for the last two digits and indicates the order of articles within the volume. Numbers start with 00, 01, 02, 03, 04, 05, 06, 07, 08, 09, OA, OB...0Z, followed by 10-1Z, 20-2Z, etc.

\author{
Ablameyko, Sergey, OY \\ Ai, Shengnan, 3E \\ An, Shengbiao, 21 \\ Andersson-Engels, Stefan, 1C, is \\ Antropov, Aleksandr, ol \\ Ashikbayeva, Zhannat, $\mathrm{OH}$ \\ Babin, Sergey, 이 \\ Beisenova, Aidana, $\mathrm{OH}$ \\ Blanc, Wilfried, $\mathrm{OH}$ \\ Cai, Chuangjian, $1 \mathrm{G}$ \\ Cai, Jianyong, $1 \mathrm{R}$ \\ Chang, Biao, 3A \\ Chen, Chaoliang, oW \\ Chen, Jiaming, $2 \mathrm{U}$ \\ Chen, Jianxin, $2 \mathrm{~N}, 2 \mathrm{O}, 2 \mathrm{Q}$ \\ Chen, Lingling, 3C \\ Chen, Mengdan, 38 \\ Chen, Shangbin, 37 \\ Chen, Shuo, $2 \mathrm{U}$ \\ Chen, Shuwang, 21 \\ Chen, Sung-Liang, $1 \mathrm{U}$ \\ Chen, Zhengyu, 3E \\ Chen, Zhiyi, $1 \mathrm{~V}$ \\ Chen, Zhongping, 25, 26 \\ Chepurna, O., $1 \mathrm{~A}$ \\ Dai, Cuixia, OR \\ Dandekar, T., IA \\ Dempsey, Eugene, 1C \\ Deng, Kexin, $1 G$ \\ Ding, Rui, 16 \\ Dobashi, Yuta, OW \\ Dong, Hongzhou, 25, 26 \\ Evmenova, Ekaterina, 0 \\ Fan, Zihui, $2 \mathrm{U}$ \\ Fang, Yuhong, 32 \\ Floris, Francesco, is \\ Frohme, M., 1 A \\ Fu, Yuting, 2G \\ Gao, Siqi, 0 N, 24 \\ Gao, Wanrong, OW \\ Gong, Wei, 0N, 32 \\ Gong, Xiaojing, 1V, 2T \\ Grebinyk, A., 1 A \\ Grebinyk, S., 1 A \\ Gu, Ying, 2D, 2X, 3A \\ Guo, Wenhui, 2N, 2O, 2Q \\ Han, Changpeng, 2K, $2 M$ \\ Han, Guang, 12 \\ Han, Shanshan, $1 \mathrm{U}$
}

Han, Xue, $1 X$

Han, Zhen, 16

Hao, Junhai, $2 Z$

$\mathrm{He}$, Bin, 3E

$\mathrm{He}, \mathrm{HaO}, 19,1 \mathrm{~N}$

$\mathrm{He}$, Jiajia, $2 \mathrm{O}$

$\mathrm{He}$, Xiaoliang, $1 \mathrm{Y}$

He, Youwu, 2L

$\mathrm{Hou}$, Jing, 3C

Hsieh, Jui-Cheng, 3E

Hu, Weiwang, 13

$\mathrm{Hu}$, Ying, $2 \mathrm{X}$

$\mathrm{Hu}$, Yong, 06

Huang, Jin, 37

Huang, Yimei, 2B, 2J

Huang, Zheng, 16, 32

Issatayeva, Aizhan, $\mathrm{OH}$

Jelbuldina, Madina, $\mathrm{OH}$

Ji, Tengda, 3A

Jiang, Zhilong, $1 Y$

Jiao, Ruonan, $1 X$

Jie, Jiang, 16

Jin, Luhong, OY

Jing, Lili, 10

Jivraj, Jamil, OW

Kablukov, Sergey, 이

Kang, Deyong, 2N, 2O, 2Q

Kharenko, Denis S., 0

Kolosova, Viktoria V., OK

Kong, Fanyi, $1 \mathrm{X}$

Kong, Huan, 2K

Kong, Ruiming, OR

Kong, Xianghan, 37

Kong, Yan, $1 Y$

Korganbayev, Sanzhar, $\mathrm{OH}$

Krite Svanberg, Emilie, 1C

Kuang, Cuifang, OY

Kuznetsov, Alexey, 이

Li, Buhong, 2D, 2X

Li, Dezi, 2L

Li, Guogiang, OR

Li, Heping, 26

Li, Hui, 13, 2 Z

Li, Hui, IR

Li, Hui, 25

$\mathrm{Li}, \mathrm{Ke}, 2 \mathrm{~L}$

Li, Lianhuang, 2N, 2O, 2Q

Li, Linxin, 2D

Li, Mingsuo, 2R 
Li, Qin, 08

Li, Renjian, 3C

Li, Weijun, 16

Li, Xi, 13

Li, Xianqiang, 15

$\mathrm{Li}, \mathrm{XU}, 2 \mathrm{~F}$

Li, Yunqi, 3A

Li, Zhifang, 1R, 2L

Liao, Wenchao, 3E

Liao, Xianglian, $2 \mathrm{X}$

Lin, Hao, $2 \mathrm{U}$

Lin, Hongxin, 32

Lin, Juqiang, ON, 24, 2B, 2J

Lin, Lisheng, $2 \mathrm{X}$

Lin, Meiai, 2A

Lin, Wanni, OY

Lin, Yamin, 24, $2 \mathrm{~J}$

Lin, Yating, 2B, $2 \mathrm{~J}$

Lin, Yongping, $1 R$

Lin, Zhanyi, $2 Z$

Liu, Ailin, OR

Liu, Cheng, $1 Y$

Liu, Chi, 3D

Liu, Fang, 12

Liu, Guodong, 22

Liu, Hua, 2R

Liu, Kun, 1X

Liu, Lina, 2D, $2 X$

Liu, Lixin, 2V

Liu, Qi, 2G

Liu, Qiao, $1 T$

Liu, Shangmin, $2 Z$

Liu, Wei, 08

Liu, Xin, OU, 2K, 2M, 3K

Liu, Yidi, 3A

Liu, Ying, $2 \mathrm{~K}$

Lu, Fake, oJ

Lu, Huihui, is

LU, Mengyang, OU

LU, Xinmin, OR

Luo, Xiaofei, IW

Lv, Xuekun, 3C

Ma, Cheng, IG

Ma, Hehe, 3K

Mai, Tongtong, $2 \mathrm{~F}$

Mao, Jiangbing, 38

Matveeva, Karina I., OK

Matyshevska, O., 1A

Misuk, Vladimir O., OK

Moiseeva, Ekaterina M., OK

Molardi, Carlo, $\mathrm{OH}$

Moon, Andy, 06

Nie, Kaixuan, 3D

Ohulchanskyy, T. Y., 1A

Pacheco, Andrea, 1C

Pellionisz, Peter A., 06

Peng, Xiao, 3D

Peng, Zhangkai, 3E

Peng, Zheng, 3D

Pensa, Jake, 06
Petrushko, Yu., $1 \mathrm{~A}$

Prylutska, S., 1A

Qin, $\mathrm{Na}, 08$

Qiu, Haixia, 3A

Qiu, Rui, OR

Qu, Junle, 1A, 3D

Rafalskiy, Vladimir $\mathrm{V}$., OK

Rangwalla, Khuzaima, 06

Ren, Wenjiao, $2 Q$

Ren, Zhong, 22

Rensing, Marc, is

Ritter, U., 1 A

Ruan, Weiwei, 38

Sales, Salvador, $\mathrm{OH}$

Samusev, llia G., OK

Schena, Emiliano, $\mathrm{OH}$

Shen, Tingfeng, 20

Shi, Weisong, OW

Shu, Chengyou, 1V, 2T

Shu, Yuexia, 2K, 3K

Song, Haiyang, $1 R$

Song, Jun, 3D

Song, Liang, 1V, 2T

Song, Lipei, 3C

Song, Yiwan, 3D

Sovetov, Sultan, $\mathrm{OH}$

Srivastava, Prateek K., $1 U$

St. John, Maie A., 06

Su, Xuantao, 1T, 2A

Su, Ya, 2F

Sun, Changsen, $1 \mathrm{X}$

Sun, Hao, 08

Sun, Jing, $1 \mathrm{~T}$

Sun, Weiming, 3C

Suo, Yuanzhen, $0 \mathrm{~J}$

Tan, Hao, $2 \mathrm{~F}$

Tang, Bihua, 3E

Tang, Shuzhen, ON, 24, 2B, 2J

Tang, Wanyi, 19

Tang, Xueli, $2 \mathrm{M}$

Tao, Lechan, 2G

Tcibulnikova, Anna V., OK

Tian, Xiaoying, $1 \mathrm{~N}$

Tian, Ye, $1 Z$

Tosi, Daniele, $\mathrm{OH}$

Tsapkova, Alina A., OK

Tu, Chang-Ching, $1 \mathrm{U}$

Wang, Bo, $1 \mathrm{~W}$

Wang, Chengming, 3E

Wang, Chuan, $2 \mathrm{~N}, 2 \mathrm{O}, 2 \mathrm{Q}$

Wang, Congcong, $1 \mathrm{~W}$

Wang, Huiquan, $1 \mathrm{Z}$

Wang, Jingbo, 08

Wang, Jinhai, $1 Z$

Wang, Lan, IT

Wang, Ping, 37

Wang, Pinghe, 25, 26

Wang, Shouyu, $1 Y$

Wang, Shu, 2N, 20

Wang, Xuanhao, $1 \mathrm{G}$ 
Wang, Ying, 2D

Wang, Yuhua, 38

Wei, Liya, 2F

Wei, Xunbin, 2G

Wen, Kai, 2V

WU, Wenjuan, OR

Wu, Xinglong, 37

Wu, Yueheng, $2 Z$

Wu, Yutong, 26

$X i$, Gangqin, $2 \mathrm{~N}$

Xiao, Jiaying, $1 \mathrm{~W}$

Xie, Shusen, ON, 24, 2B, 2J, 32

Xie, Xiaoliang Sunney, $0 \mathrm{~J}$

Xie, Zhihua, 1V, 2T

$X U$, Jianshu, 2B, 2J

$X \cup$, Liu, OY

$X U$, Yingke, $O Y$

Xue, Ping, 2V, 3E

Yan, Shuai, 37

Yang, Daya, $1 \mathrm{~V}$

Yang, Deyuan, 32

Yang, Hongqin, 38

Yang, Qianqian, 2V

Yang, Victor X. D., OW

Yang, Wenlong, 0J

Yang, Wenzhao, $1 U$

Yao, X. Steve, 2F

Yashchuk, V. M., IA

Yu, Jia, 13, $2 Z$

Yu, Yun, ON, 24

Yu, Yunfei, $2 X$

Yuan, Yufeng, 3D

Zhang, $\mathrm{He}, 2 \mathrm{U}$

Zhang, Li-Peng, 15

Zhang, Meiling, $2 \mathrm{~V}$

Zhang, Ning, 3E

Zhang, Qinke, 2U

Zhang, Rongli, 13, 2 Z

Zhang, Shuyu, 2A

Zhang, Wenxin, 3E

Zhang, $X i, 1 Y$

Zhang, Xiao, 3E

Zhao, Chengwei, 2 I

Zhao, Fenqiang, OY

Zhao, Hongwei, OR

Zhao, Hongyou, 3A

Zhao, Yuxia, 15

Zheng, Liqin, 2N, 2O, 32

Zheng, Mengmeng, ON, 24, 2B, 2J

Zheng, Mingjie, $2 \mathrm{~L}$

Zheng, Rongsheng, $1 \mathrm{R}$

Zheng, Wei, 13, 2 Z

Zhou, Enze, 37

Zhou, Liwei, $2 \mathrm{R}$

Zhou, Quanyu, 2G

Zhou, Tianyang, OU

Zhou, Xiaoxu, OY

Zhu, Ronggang, $2 R$

Zou, Jian, 16

Zyubin, Andrey Y., OK 
Proc. of SPIE Vol. $111901119001-12$

Downloaded From: https://www.spiedigitallibrary.org/conference-proceedings-of-spie on 26 Apr 2023 Terms of Use: https://www.spiedigitallibrary.org/terms-of-use 


\title{
Symposium Committees
}

\author{
General Chairs
}

Jim M. Oschmann, President, SPIE and Ball Aerospace (United States)

Qihuang Gong, President, Chinese Optical Society and Peking University (China)

General Co-chairs

Guangcan Guo, Past President, Chinese Optical Society and University of Science and Technology of China (China)

Zejin Liu, Vice President, Chinese Optical Society and National University of Defense Technology (China)

Technical Program Chairs

Ruxin Li, Vice President, Chinese Optical Society and Shanghai Institute of Optics and Fine Mechanics (China)

Xingde Li, Johns Hopkins University (United States)

Technical Program Co-chairs

Tianchu Li, National Institute of Metrology (China)

Wei Huang, Northwestern Polytechnical University (China)

Ying Gu, Vice President, Chinese Optical Society and PLA General Hospital (China)

Huilin Jiang, Changchun University of Science and Technology (China)

Wenqing Liu, Vice President, Chinese Optical Society, and Anhui Institute of Optics and Fine Mechanics (China)

Guobin Fan, China Academy of Engineering Physics (China)

Suotang Jia, Vice President, Chinese Optical Society, and Shanxi University (China)

Xiaomin Ren, Vice President, Chinese Optical Society, and Beijing University of Posts and Telecommunications (China)

Secretaries-General

Bo Gu, Deputy Secretary General, Chinese Optical Society (China)

Hong Yang, Deputy Secretary General, Chinese Optical Society and Peking University (China) 
Yan Li, Deputy Secretary General, Chinese Optical Society, and Peking University (China)

Daoxin Dai, Zhejiang University (China)

Local Organizing Committee Chair

Xu Liu, Secretary General, Chinese Optical Society and Zhejiang University (China)

Local Organizing Committee Co-chairs

Jianrong Qiu, Zhejiang University (China)

Daoxin Dai, Zhejiang University (China)

Local Secretaries

Wei Xiong, Chinese Optical Society (China)

Qing Yang, Zhejiang University (China)

Local Organizing Committee

Qing Yang, Zhejiang University (China)

Lan Wu, Zhejiang University (China)

Yaocheng Shi, Zhejiang University (China)

Dong Liu, Zhejiang University (China)

Yungui Ma, Zhejiang University (China)

Ke Si, Zhejiang University (China)

Yang Yang, Zhejiang University (China)

Xinyong Dong, China Jiliang University (China)

Le Wang, China Jiliang University (China)

Fei Tong, Chinese Optical Society (China)

Technical Organizing Committee

Mohammad Hossein Asghari, Loyola Marymount University

(United States) and Tachyonics Inc. (United States)

Pablo Benítez, Universidad Politécnica de Madrid (Spain)

Liangcai Cao, Tsinghua University (China)

P. Scott Carney, University of Rochester (United States)

Benyong Chen, Zhejiang University of Science and Technology

(China)

Hongqiang Chen, GE Global Research (United States)

Daoxin Dai, Zhejiang University (China)

Qionghai Dai, Tsinghua University (China)

Qihuang Gong, Peking University (China)

Ying Gu, Chinese PLA General Hospital (China) 
Guang-Can Guo, University of Science and Technology of China (China)

Byoung Seung Ham, Gwangju Institute of Science and Technology (Korea, Republic of)

Sen Han, University of Shanghai for Science and Technology (China) and Suzhou H\&L Instruments, LLC (China)

Zuyuan He, Shanghai Jiao Tong University (China)

Werner H. Hofmann, Technische Universität Berlin (Germany)

Minghui Hong, National University of Singapore (Singapore)

Bahram Jalali, University of California, Los Angeles (United States)

Satoshi Kawata, Osaka University (Japan)

Baojun Li, Jinan University (China)

Ming Li, Institute of Semiconductors, CAS (China)

Ruxin Li, Shanghai Institute of Optics and Fine Mechanics (China)

Xingde Li, Johns Hopkins University (United States)

Jian Liu, PolarOnyx, Inc. (United States)

Tiegen Liu, Tianjin University (China)

Yongfeng Lu, University of Nebraska-Lincoln (United States)

Qingming Luo, Huazhong University of Science and Technology (China)

Gang-Ding Peng, The University of New South Wales (Australia)

Osamu Matoba, Kobe University (Japan)

Min Qiu, Westlake University (China)

Yuji Sano, ImPACT (Japan)

Yunlong Sheng, Université Laval (Canada)

Kebin Shi, Peking University (China)

Tsutomu Shimura, The University of Tokyo (Japan)

Upendra N. Singh, NASA Langley Research Center (United States)

Michael G. Somekh, Shenzhen University (China)

Yuguo Tang, Suzhou Institute of Biomedical Engineering and

Technology (China)

Masahiko Tani, University of Fukui (Japan)

Limin Tong, Zhejiang University (China)

Kazumi Wada, Massachusetts Institute of Technology (United States)

Yongtian Wang, Beijing Institute of Technology (China)

Rongshi Xiao, Beijing University of Technology (China)

Hongxing $\mathbf{X u}$, Wuhan University (China)

Jianhua Yao, Zhejiang University of Technology (China)

Toru Yoshizawa, Tokyo University of Agriculture and Technology (Japan) and 3D Associates, Inc. (Japan)

Changyuan Yu, The Hong Kong Polytechnic University

(Hong Kong, China)

Xiao-Cong Yuan, Shenzhen University (China)

Cunlin Zhang, Capital Normal University (China)

Song Zhang, Purdue University (United States)

Xi-Cheng Zhang, University of Rochester (United States) 
Xinliang Zhang, Wuhan National Laboratory for Optoelectronics

(China)

Xuping Zhang, Nanjing University (China)

Zhenrong Zheng, Zhejiang University (China)

Changhe Zhou, Shanghai Institute of Optics and Fine Mechanics (China)

Zhiping Zhou, Peking University (China)

Dan Zhu, Huazhong University of Science and Technology (China)

Ning Hua Zhu, Institute of Semiconductors, CAS (China) 


\title{
Conference Committee
}

\author{
Symposium Chairs
}

Jacobus M. Oschmann, Ball Aerospace (United States)

Qihuang Gong, Peking University (China)

Conference Chairs

Qingming Luo, Hainan University (China)

Xingde Li, Johns Hopkins University (United States)

Ying Gu, Chinese PLA General Hospital (China)

Yuguo Tang, Suzhou Institute of Biomedical Engineering and Technology (China)

Conference Co-chair

Dan Zhu, Huazhong University of Science and Technology (China)

Conference Program Committee

Jing Bai, Tsinghua University (China)

Stephen A. Boppart, University of Illinois (United States)

Shih-Chi Chen, The Chinese University of Hong Kong (Hong Kong, China)

Wei R. Chen, University of Central Oklahoma (United States)

Yu Chen, University of Maryland, College Park (United States)

Linhong Deng, Chongqing University (China)

Zhihua Ding, Zhejiang University (China)

Qiyong Gong, West China Hospital (China)

Hui Li, Fujian Normal University (China)

Hong Liu, The University of Oklahoma (United States)

Huafeng Liu, Zhejiang University (China)

Hui Ma, Tsinghua University (China)

Yingtian Pan, Stony Brook University (United States)

Paras N. Prasad, University at Buffalo (United States)

Jun Qian, Zhejiang University (China)

Yuwen Qin, National Natural Science Foundation (China)

Junle Qu, Shenzhen University (China)

Kebin Shi, Peking University (China)

Ke Si, Zhejiang University (China)

Jie Tian, Institute of Automation (China)

Valery V. Tuchin, Saratov State University (Russian Federation)

Lihong V. Wang, California Institute of Technology (United States) and Washington University in St Lovis (United States) 
Ruikang K. Wang, University of Washington (United States)

Xueding Wang, University of Michigan (United States)

Xunbin Wei, Shanghai Jiao Tong University (China)

Xujie Xia, Shanghai Jiao Tong University (China)

Da Xing, South China Normal University (China)

Kexin Xu, Tianjin University (China)

Xibin Yang, Suzhou Institute of Biomedical Engineering and Technology (China)

Yudong Zhang, Institute of Optics and Electronics (China)

Zhenxi Zhang, Xi'an Jiaotong University (China)

\section{Session Chairs}

1 Translational Optical Techniques for Clinical Medicine I

Ying Gu, Chinese PLA General Hospital (China)

2 Translational Optical Techniques for Clinical Medicine II

Yanmei Liang, Nankai University (China)

3 Nanobiophotonics and Sensors

Qingming Luo, Hainan University (China)

4 Biomedical Spectroscopy

Zhongze Gu, Southeast University (China)

5 Advanced Optical Imaging Techniques

Hui Ma, Graduate School at Shenzhen, Tsinghua University (China)

Wei Gong, Fujian Normal University (China)

6 Optical Theranostics I

Dan Zhu, Huazhong University of Science and Technology (China)

7 Optical Theranostics II

Haixia Qiu, Chinese PLA General Hospital (China)

8 Tissue Optics/Laser-Tissue Interaction

Buhong Li, Fujian Normal University (China)

9 Multimodal Biomedical Imaging

Qin Li Sr., Beijing Institute of Technology (China) 